\title{
A Dilated Trigonometrically Equipped Algorithm to Compute Periodic Vibrations through Block Milne's Implementation
}

\author{
J. G. Oghonyon \\ Department of Mathematics \\ Covenant University \\ Ota, Nigeria \\ godwin.oghonyon@ \\ covenantuniversity.edu.ng
}

\author{
S. A. Bishop \\ Department of Mathematics \\ Covenant University \\ Ota, Nigeria \\ sheila.bishop@, \\ covenantuniversity.edu.ng
}

\author{
K. S. Eke \\ Department of Mathematics \\ Covenant University \\ Ota, Nigeria \\ kanayo.eke@ \\ covenantuniversity.edu.ng
}

\begin{abstract}
This paper intends to investigate the use of a dilated trigonometrically equipped algorithm to compute periodic vibrations in block Milne's implementation. The block-Milne implementation is established by developing a block variablestep-size predictor-corrector method of Adam's family using a dilated trigonometrically equipped algorithm. The execution is carried out using a block variable-step-size predictor-corrector method. This system has significant advantages that include the varying step-size and finding out the convergence-criteria and error control. Convergence-criteria and operational mode are discussed to showcase the accuracy and effectuality of the proposed approach.
\end{abstract}

Keywords-dilated trigonometrically equipped algorithm; blockMilne's device; convergence-criteria; max errors; principal-localtruncation-error

\section{NOMENCLATURE}

BMD: errors in BMD for computing test problems 1, 2 and 3.

$\mathrm{M}_{\text {employed: }}$ approach employed.

Max $_{\text {errors: }}$ the magnitude of the max errors of BMD.

$C_{\text {criteria }}$ : convergence-criteria.

TSDM: errors in TSDM (trigonometrically-fitted Second derivative method) for numerical tested problem 1, [12].

BHMTB: errors in BHMTB (block hybrid method with trigonometric basis) for numerical tested problem $1[10]$.

BHT: errors in BHT (block hybrid trigonometrically fitted of $\delta=10^{-6}$ ) for numerical tested problem 1,2 and 3 [13].

BHTRKNM: errors in BHTRKNM (block hybrid trigonometrically fitted Runge-Kutta-Nystrom method of $\delta=10^{-6}$ ) for numerical tested problem 1,2 and 3 [14].

BHTFM: errors in BHTFM (block hybrid trigonometrically fitted method) for numerical tested problem $2[11]$

\section{INTRODUCTION}

The dilated trigonometrically equipped algorithm is virtually among the utilitarian procedures which constitute block-Milne's implementation for approximating solutions of periodic vibrations. A trigonometrically equipped algorithm is more effective compared to non-fitted methods especially when the outcome exhibits periodical vibrations $[1,2]$. The Block
Milne's implementation is of significant importance for documneting block variable-step-size predictor-corrector methods, convergence-criteria and check errors [3-6]. This composition aims at approximating the solution of periodic vibrations of class: $[1,6]$

$$
\begin{aligned}
& g^{\prime \prime}=f(v, g), g\left(v_{0}\right)=g_{0}, g^{\prime}\left(v_{0}\right)=g_{0}{ }^{\prime} \\
& \text { for } v \in\left[v_{0}, X\right]
\end{aligned}
$$

where $f: R \times R^{c} \rightarrow R^{c}, c$ is the dimension of the physical system. Presuming $\mathrm{f} \in \mathrm{R}$ equals to the differentiability to sufficient degree on $\mathrm{u} \epsilon\left[v_{0}, X\right]$ and meets a worldwide Lipchitz precondition, i.e., $L \geq 0 \ni$

$$
\mid f(v, g)-f(v, \bar{g}|\leq L| g-\bar{g} \mid, \forall g, \bar{g} \in R .
$$

Underneath this presumption, (1) ascertains the universal and singularity place as $v \in[u, \rho]$ which is similarly considered to meet the Weierstrass theorem [6-9]. In particular, periodic vibrations often spring up in areas of scientific knowledge like Newtonian mechanics, uranology, quantum theory, control theory, electric circuits and biological science. Various approximation methods have been established. Clear cut methods instituted on trigonometrically equipped algorithms may be realized [1, 10-18]. Their execution is carried out while adopting fixed step-size strategies [1, 10-22]. The motive governing a trigonometrically equipped algorithm is inherent in the fact that if the frequency is acknowledged in advance, this method turns more beneficial compared to the multinomial established methods [1, 10-18].

In addition, a block Milne's implementation is primarily employed for changing step-size, fixing convergence-criteria and check ciphered errors [3-6, 23-26]. The main target of this paper is to implement a dilated trigonometrically equipped algorithm for ciphering periodic vibrations where the frequency of the outcome is anticipated in advance. This approach possesses advantages like varying step-size, deciding the convergence criteria and error control [7-8, 24-25, 27-29]. The dilated trigonometrically equipped algorithm has extra advantages which includes reduced computation costs and 
improved accuracy. Milne's implementation is examined as a broad prospect of the predictor-corrector method on account of the computational benefits. This approach employs components like Adams type class, block predictor-corrector pair of similar order and principal local truncation errors as mentioned in [7-8, 24-25, 27-29].

Definition 1: $d-$ block, $b$ - stage method. Whenever $k$ refers to block size and $h$ as pace size, then block size in time is $b h$. Let $m=0,1,2, \ldots$ form the block number and let $t=m b$, then the $d$-block, $b$ - point method can be composed in the next class:

$$
Y_{\tau}=\sum_{\mu=1}^{d} A_{\mu} Y_{\tau-\mu}+h \sum_{\mu=0}^{d} B_{\mu} F_{\tau-\mu}
$$

$$
\text { For } Y_{t}=\left[y_{t+1}, \ldots, y_{t+i}, \ldots, y_{t+r}\right]^{\tau}, F_{t}=\left[f_{t+1}, \ldots, f_{t+i}, \ldots, f_{t+r}\right]^{\tau}
$$

$A_{\mu}$ and $B_{\mu}$ are $b \times b$ constants matrices [6, 17, 30]. Hence, taking off from the over account, a block method has computational benefits. For each practical real-life program, the terminate product is assessed to a greater extent at the same time. Utilizing these methods can permit faster outcomes of the problem which can be handled to generate the sought after accuracy [10-12, 16-17].

\section{METHODS}

Block-Milne's device is a combination of Adams-Bashforth $g$-step (predictor) method and Adams-Moulton $g-1-$ step (corrector) method of the same order. This combination can be of the form:

$$
\begin{aligned}
& g(v)=\sum_{l=0}^{k} \alpha_{l} g_{t-l}+h^{2} \sum_{l=0}^{k} \beta_{l}(\delta) f_{t-l}, \\
& g(v)=\sum_{l=0}^{k} \alpha_{l} g_{t-l}+h^{2} \sum_{l=1}^{k} \boldsymbol{\beta}_{l}^{*}(\delta) f_{t+l} .
\end{aligned}
$$

Equations (3) and (4) represent the Adams family of block Milne's device with $\delta=w h, \beta_{n}(\delta), n=0,1,2$ comprising invariants that depend on the varying step-size and frequency. Observing that $g_{t+l}$ is the numeric approximate of the analytical results $g\left(v_{t+l}\right)$ i.e. $g\left(v_{t+l}\right) \approx g_{t+l}$, and $f_{t+n} \approx$ $f\left(v, g_{t+n}\right)$ owning $n=0,1,2$. To arrive at (3) and (4), the trigonometrically fitted method is written as the dilated trigonometrically equipped algorithm in which runs by looking forward to approximate the analytical result $g(v)$ on distinct time intervals of $\left[v_{t}, v_{t-j}\right]$ through with the interpolating subprogram of (5):

$g(v)=\propto_{0}+\propto_{1} v+\propto_{2} v^{2}+\propto_{3} \sin (w v)+\propto_{4} \cos (w v)$

Revising (4) produces the dilated trigonometrically equipped algorithm as:

$$
\begin{aligned}
& g(v)=\propto_{0}+\propto_{1}\left(\frac{v-v_{n}}{h}\right)+\propto_{2}\left(\frac{v-v_{n}}{h}\right)^{2}+\propto_{3}\left(\mathrm{w}\left(\frac{v-v_{\mathrm{n}}}{\mathrm{h}}\right)-\frac{\mathrm{w}^{3}}{6}\left(\frac{v-v_{\mathrm{n}}}{\mathrm{h}}\right)^{3}\right)+ \\
& \propto_{4}\left(1-\frac{w^{2}}{2}\left(\frac{v-v_{t}}{h}\right)^{2}+\frac{w^{4}}{24}\left(\frac{v-v_{t}}{h}\right)^{4}\right),
\end{aligned}
$$

where $\propto_{0}, \propto_{1}, \propto_{2}, \propto_{3}$ and $\propto_{4}$ are invariants needed to be determine $\delta$ in a especial direction. Presume the precondition that (6) matches with the accuracy output at definite length of time $v_{t}, v_{t-n}$ to get the approximant of (7):

$$
y\left(v_{t}\right) \approx y_{t}, \quad y\left(v_{t-n}\right) \approx y_{t-n} .
$$

Demanding that the interpolating function (6) conforms to (1) at the levels $v_{i+k}, k=0,1,2$ we get the next approximates as

$$
g^{\prime}\left(v_{t+k}\right) \approx f_{t+k}, g^{\prime \prime\left(v_{t+k}\right)} \approx f_{t+k}, k=0,1,2
$$

Linking (7) and (8) will lead to a fivefold system of equations which gives birth to $\mathrm{Av}=\mathrm{d}$. Computing the systems of equation adopting Mathematica 9 kernel 64 to obtain $v_{k}, k=0,1,2,3,4$ and subbing the measures of $v_{k}{ }^{\prime} s$ into (6) will yield the continuous block Milne's device. Assessing the continuous block Milne's device at some preferred points of $v_{t+k}, k=1$, 2, 3 will invent the Milne's device as

$g(v)=g_{l}+g_{l-1}+h^{2}\left(\beta_{1}(w, v) f_{l+1}+\beta_{2}(w, v) f_{l+2}+\beta_{3}(w, v) f_{l+3}\right)(9)$

where $w$ is the frequency, and $\beta_{1}(w, v), \beta_{2}(w, v)$, and $\beta_{3}(w, v)$ are uninterrupted invariants $[5,10-14]$.

\section{A. Forming Convergence Criteria for Block Milne's Device}

In launching operation of block-Milne's device, AdamsBashforth $g$-step and Adams-Moulton $g-1-$ step approaches are employed in a predictor-corrector approach [36, 23-25]. Mixed block-Milne's device proves the feasibleness to determine principal-local-truncation-error in predictorcorrector approach in absence of finding higher differential coefficient, $g(v)$. Taking for granted that $\tilde{q}=\bar{q}$, where $\bar{q}$ and $\tilde{q}$ showcase the order of the predictor and corrector. Right now, in approach of order $\tilde{q}$, the investigation of the block-AdamsBashforth $g$-step brings forth principal-local-truncationerrors.

$\bar{C}_{q+5}^{[1]} h^{q+5} g^{(q+5)}\left(\bar{v}_{n}\right)=g\left(v_{l+1}\right)-g_{l+1}^{\left[i_{1}\right]}+O\left(h^{q+6}\right)$,
$\tilde{C}_{q+5}^{[2]} h^{q+5} y^{(q+5)}\left(\tilde{v}_{n}\right)=y\left(v_{l+2}\right)-g_{l+2}^{\left[i_{2}\right]}+O\left(h^{q+6}\right)$
$\tilde{C}_{q+5}^{[3]} h^{p+5} g^{(p+5)}\left(\tilde{v}_{n}\right)=g\left(v_{l+3}\right)-g_{l+3}^{\left[i_{3}\right]}+O\left(h^{q+6}\right)$.

A corresponding break down of block-Adams-Moulton $g-1-$ step brings about principal-local-truncation-errors:

$$
\begin{gathered}
\bar{W}_{q+5}^{[1]} h^{q+5} g^{(q+5)}\left(\bar{v}_{n}\right)=g\left(v_{l+1}\right)-g_{l+1}^{\left[r_{1}\right]}+O\left(h^{q+6}\right), \\
\bar{W}_{q+5}^{[2]} h^{q+5} g^{(q+5)}\left(\bar{v}_{n}\right)=g\left(v_{l+2}\right)-g_{l+2}^{\left[r_{2}\right]}+O\left(h^{q+6}\right) \\
\bar{W}_{q+5}^{[3]} h^{q+5} g^{(q+5)}\left(\bar{v}_{n}\right)=g\left(v_{l+3}\right)-g_{l+3}^{\left[r_{3}\right]}+O\left(h^{q+6}\right),
\end{gathered}
$$

where $\tilde{C}_{q+5}^{[1]}, \tilde{C}_{q+5}^{[2]}, \tilde{C}_{q+5}^{[3]}, \bar{W}_{q+5}^{[1]}, \bar{W}_{q+5}^{[2]}$ and $\bar{W}_{q+5}^{[3]}$ are existing as independent entities of the step-size $\mathrm{h}$ and $g(v)$ behaves as the result to the differential coefficient fulfiliing the initial consideration $g\left(v_{n}\right) \approx g_{n}[3-6,23-25]$. To move ahead, the precondition for small assesses of $\mathrm{h}$ is reached as $g^{(5)}\left(\tilde{v}_{n}\right) \approx$ $g^{(5)}\left(\bar{v}_{n}\right)$, and the dominance of block-Milne's device banks right away on this condition. Further simplification of the principal local truncation errors of (10) and (11) while neglecting terms of $O\left(h^{q+6}\right)$ order, makes it easy to achieve computation of principal- local-truncation-errors of blockMilne's device as:

$$
\begin{aligned}
& \bar{W}_{q+5}^{[1]} h^{q+5} g^{(q+5)}\left(\bar{v}_{n}\right) \approx \frac{\bar{W}_{q+5}^{[1]}}{\tilde{\tilde{C}}_{q+5}^{[1]}-\bar{W}_{q+5}^{[1]}}\left[g_{n+l}^{\left[i_{1}\right]}-g_{n+l}^{\left[r_{1}\right]}\right]<\omega_{1}, \\
& \bar{W}_{q+5}^{[2]} h^{q+5} g^{(q+5)}\left(\bar{v}_{n}\right) \approx \frac{\bar{W}_{q+5}^{[2]}}{\tilde{C}_{q+5}^{[2]}-\bar{W}_{q+5}^{[2]}}\left[g_{n+l}^{\left[i_{2}\right]}-g_{n+l}^{\left[r_{2}\right]}\right]<\omega_{2},
\end{aligned}
$$


$\bar{W}_{q+5}^{[3]} h^{q+5} g^{(q+5)}\left(\bar{v}_{n}\right) \approx \frac{\bar{W}_{q+5}^{[3]}}{\tilde{C}_{q+5}^{[3]}-\bar{W}_{q+5}^{[3]}}\left[g_{n+l}^{\left[i_{3}\right]}-g_{n+l}^{\left[r_{3}\right]}\right]<\omega_{3}$.

Mentioning the affirmations that $g_{n+l}^{\left[i_{1}\right]} \neq g_{n+l}^{\left[r_{1}\right]}, g_{n+l}^{\left[i_{2}\right]} \neq g_{n+l}^{\left[r_{2}\right]}$ and $g_{n+l}^{\left[i_{3}\right]} \neq g_{n+l}^{\left[r_{3}\right]}$ are the predicted and corrected approximates which are given by the block Milne's device of order $\mathrm{p}$, while $\bar{W}_{q+5}^{[j]} h^{q+5} g^{(q+5)}\left(\bar{v}_{n}\right), j=1,2,3$ are the principal-localtruncation-errors. $\omega_{1}, \omega_{2}$ and $\omega_{3}$ are the boundaries of the convergence-criteria of block-Milne's device. Moreover, the estimates of the principal local truncation error (12) ar utilized to determine whether to go for the results of the current step or to reconstruct the step with a slighter variable-step-size. The step is established on a trial run as defined by (12) [3-6, 23-25]. The principal-local-truncation-error (12) is the convergencecriteria of block-Milne's, device distinctly seen as blockMilne's device (estimate) for conforming to convergence.

\section{NUMERICAL EXAMPLES}

Three selected numerical test problems were viewed and solved utilizing the proposed approach (denoted as BMD in this paper) at different convergence criteria of $10^{-3}, 10^{-5}$, $10^{-7}, 10^{-9}$ and $10^{-11}$ [10-14]. Programming codes on block Milne's are compiled employing Mathematica 9 kernel 64 . These codes are executed in a block by block mode together with the block Milne's device.

Numerical test problem 1. Consider the inhomogeneous IVP:

$y^{\prime \prime}(v)=-100 y+99 \sin (v), y(0)=1, y^{\prime}(0)=11$, $0 \leq v \leq 1000$.

Solution:

$$
y(v)=\cos (10 v)+\sin (10 v)+\sin (v)
$$

Numerical test problem 2. Consider the nonlinear Duffing equation: $y^{\prime \prime}+y+y^{3}=B \cos (\Omega v), y(0)=C_{0}, y^{\prime}(0)=0$.

Solution:

$y(v)=C_{1} \cos (\Omega v)+C_{2} \cos (3 \Omega v)+C_{3} \cos (5 \Omega v)+$ $C_{4} \cos (7 \Omega v)$ where $\Omega=1.0, \quad B=2 \times 10^{-3}, \quad C_{0}=0.200426728069$, $C_{1}=0.20017947753, C_{2}=0.246946143 \times 10^{-3}, C_{3}=0.304016 \times 10^{-6}$, $C_{4}=0.374 \times 10^{-9}$. Choose $w=1.01$

Numerical test problem 3. Consider the harmonic oscillator with frequency $\Omega$ and small perturbation $\delta$.

$$
\begin{array}{r}
y^{\prime \prime}+\delta y^{\prime}+\Omega^{2} y=0, \\
y(0)=0, y^{\prime}(0)=-\frac{\delta}{2}, 0 \leq v \leq 1000 .
\end{array}
$$

Solution: $y(x)=e^{\left(\frac{\delta}{2}\right) v} \cos \left(\Omega^{2}-\frac{\delta^{2}}{4}\right)$, where $\Omega=1$ and $\delta=10^{-6}$.

\section{RESUlTS}

The numerical results of the implementation of the dilated trigonometrically equipped algorithm on block Milne's device

\begin{tabular}{|c|c|c|}
\hline $\mathbf{M}_{\text {employed }}$ & Max $_{\text {errors }}$ & $\mathbf{C}_{\text {criteria }}$ \\
\hline TSDM & $1.7 \mathrm{e}-03$ & $10^{-3}$ \\
\hline BHTFM & $1.2 \mathrm{e}-03$ & \\
\hline BHT & $1.9 \mathrm{e}-03$ & \\
\hline BHTRKKNM & $2.14 \mathrm{e}-03$ & \\
\hline BMD & $9.15202 \mathrm{e}-04$ & $10^{-3}$ \\
\hline BMD & $2.79178 \mathrm{e}-04$ & \\
\hline BMD & $1.83035 \mathrm{e}-04$ & \\
\hline TSDM & $2.7 \mathrm{e}-05$ & $10^{-5}$ \\
\hline BHTFM & $1.4 \mathrm{e}-05$ & \\
\hline BHMTB & $3.9 \mathrm{e}-05$ & \\
\hline BHTRKKNM & $5.98 \mathrm{e}-05$ & \\
\hline BHTRKKNM & $2.06 \mathrm{e}-05$ & \\
\hline BMD & $1.05006 \mathrm{e}-07$ & $10^{-5}$ \\
\hline BMD & $3.75372 \mathrm{e}-08$ & \\
\hline BMD & $3.34795 \mathrm{e}-06$ & \\
\hline TSDM & $1.0 \mathrm{e}-07$ & $10^{-7}$ \\
\hline BHTFM & $1.5 \mathrm{e}-07$ & \\
\hline BHMTB & $1.4 \mathrm{e}-07$ & \\
\hline BMD & $1.05933 \mathrm{e}-10$ & $10^{-7}$ \\
\hline BMD & $3.76048 \mathrm{e}-11$ & \\
\hline BMD & $3.4074 \mathrm{e}-09$ & \\
\hline TSDM & $6.3 e-09$ & $10^{-9}$ \\
\hline BHTFM & $8.7 \mathrm{e}-09$ & \\
\hline BHTFM & $1.1 \mathrm{e}-09$ & \\
\hline BHTRKKNM & $4.67 \mathrm{e}-09$ & \\
\hline BMD & $1.07025 \mathrm{e}-13$ & $10^{-9}$ \\
\hline BMD & $3.9968 \mathrm{e}-14$ & \\
\hline BMD & $3.40017 \mathrm{e}-12$ & \\
\hline BHT & $9.7 \mathrm{e}-11$ & $10^{-11}$ \\
\hline BHT & $6.7 \mathrm{e}-11$ & \\
\hline BMD & $4.88498 \mathrm{e}-15$ & $10^{-11}$ \\
\hline BMD & $1.04361 \mathrm{e}-14$ & \\
\hline BMD & $3.57492 \mathrm{e}-14$ & \\
\hline BHT & $4.3 \mathrm{e}-13$ & $10^{-13}$ \\
\hline BMD & $1.9984 \mathrm{e}-15$ & $10^{-13}$ \\
\hline BMD & $5.10703 e-15$ & \\
\hline BMD & $2.39808 \mathrm{e}-14$ & \\
\hline TSDM & $3.3 \mathrm{e}-03$ & $10^{-3}$ \\
\hline BHTFM & $1.3 \mathrm{e}-03$ & \\
\hline BMD & $3.60282 \mathrm{e}-06$ & $10^{-3}$ \\
\hline BMD & $7.81099 \mathrm{e}-06$ & \\
\hline BMD & $1.31395 \mathrm{e}-04$ & \\
\hline TSDM & $6.4 \mathrm{e}-05$ & $10^{-5}$ \\
\hline BHTFM & $5.6 \mathrm{e}-05$ & \\
\hline BHT & $7.7 \mathrm{e}-05$ & \\
\hline BHTRKKNM & $7.52 \mathrm{e}-05$ & \\
\hline BMD & $3.11695 \mathrm{e}-10$ & $10^{-5}$ \\
\hline BMD & $7.32379 \mathrm{e}-10$ & \\
\hline BMD & $1.20413 \mathrm{e}-08$ & \\
\hline TSDM & $1.0 \mathrm{e}-07$ & $10^{-7}$ \\
\hline BHTFM & $1.4 \mathrm{e}-07$ & \\
\hline BHTRKKNM & $1.34 \mathrm{e}-07$ & \\
\hline BMD & $3.14471 \mathrm{e}-14$ & $10^{-7}$ \\
\hline BMD & $7.38853 \mathrm{e}-14$ & \\
\hline BMD & $1.20062 \mathrm{e}-12$ & \\
\hline BHTRKKNM & $8.11 \mathrm{e}-09$ & $10^{-9}$ \\
\hline BMD & $1.38778 \mathrm{e}-16$ & $10^{-9}$ \\
\hline BMD & $3.60822 \mathrm{e}-16$ & \\
\hline BMD & $1.66533 \mathrm{e}-15$ & \\
\hline BHT & $1.23 \mathrm{e}-11$ & $10^{-11}$ \\
\hline BHTRKKNM & $7.13 \mathrm{e}-11$ & \\
\hline BMD & $4.06231 \mathrm{e}-13$ & $10^{-11}$ \\
\hline BMD & $9.65783 \mathrm{e}-13$ & \\
\hline BMD & $4.14513 \mathrm{e}-12$ & \\
\hline
\end{tabular}
for ciphering periodic vibrations are exhibited in Table I.
TABLE I. MILNE'S IMPLEMENTATION 


\section{CONCLUSION}

Computed results demonstrated that the BMD is attained with the aid of the convergence-criteria. These convergence criteria decide whether the result is accepted or the algorithm is repeated. The results also establish that the performance of the BMD allows improved maximum errors compared with the TSDM, BHMTM, BHT, BHTRKNM and BHTFM at all examined convergence criteria of $10^{-3}, 10^{-5}, 10^{-7}, 10^{-9}$, $10^{-11}$ and $10^{-13}$ [10-14]. Thus, it can be concluded that the devised method is suitable for working out periodic vibrations dealing with non-stiff and stiff ODEs. BMD performs better when compared to the existing methods for reasons pointed out above. Further work will deal with carrying out the block Milne's device on dilated exponentially fitted algorithm.

\section{ACKNOWLEDGMENT}

Authors would like to thank the Covenant University for providing the financial backing throughout the study time period.

\section{REFERENCES}

[1] R. D'Ambrosio, E. Esposito, B. Paternoster, "Exponentially fitted twostep hybrid methods for $\mathrm{y}^{\prime \prime}=\mathrm{f}(\mathrm{x}, \mathrm{y})$ ", Journal of Computational and Applied Mathematics, Vol. 235, No. 16, pp. 4888-4897, 2011

[2] H. Ramos, J. Vigo-Aguiar, "On the frequency choice in trigonometrically fitted methods", Applied Mathematics Letters, Vol. 23, No. 11, pp. 1378-1381, 2010

[3] U. M. Ascher, L. R. Petzold, Computer Methods for Ordinary Differential Equations And Differential Algebraic Equations, SIAM, Philadelphia, 1998

[4] J. R. Dormand, Numerical Methods for Differential Equations, CRC Press, 1996

[5] J. D. Faires, R. L. Burden, Initial-value problems for ODEs, Dublin City University, 2012

[6] J. D. Lambert, Computational Methods in mOrdinary Differential Equations, John Wiley and Sons, 1973

[7] E. Hairer, G. Wanner, Solving Ordinary Differential Equations II, Springer, 1996

[8] M. K. Jain, S. R. K. Iyengar, R. K. Jain, Numerical Methods for Scientific and Engineering Computation, New Age International, 2003

[9] L. Xie, H. Tian, "Continuous parallel block methods and their applications", Applied Mathematics and Computation, Vol. 241, pp. 356- 370, 2014

[10] F. F. Ngwane, S. N. Jator, "Block hybrid method using trigonometric basis for initial value problems with oscillating solutions", Numerical Algorithms, Vol. 63, No. 4, pp. 713-725, 2013

[11] F. F. Ngwane, S. N. Jator, "Solving oscillatory problems using a block hybrid trigonometrically fitted method with two off-step points. Electronic Journal of Differential Equations", 9th Conference on Differential Equations and Computational Simulations, Vol. 20, pp. 119132,2013

[12] F. F. Ngwane, S. N. Jator, "Trigonometrically-fitted second derivative method for oscillatory problems", Springer Plus, Vol. 3, No. 1, 2014

[13] F. F. Ngwane, S. N. Jator, "Solving the telegraph and oscillatory differential equations by a block hybrid trigonometrically fitted algorithm", International Journal of Differential Equations, Vol. 2015, Article ID 347864, 2015

[14] F. F. Ngwane, S. N. Jator, "A trigonometrically fitted block method for solving oscillatory second-order initial value problems and Hamiltonian systems", International Journal of Differential Equations, Vol. 2017, Article ID 9293530, 2017
[15] G. Psihoyios, T. E. Simos, "Trigonometrically fitted predictor-corrector methods for IVPs with oscillatory solutions", Journal of Computational and Applied Mathematics, Vol. 158, No. 1, pp. 135-144, 2003

[16] G. Psihoyios, T. E. Simos, "A fourth algebraic order trigonometrically fitted predictor-corrector scheme for IVPs with oscillating solutions", Journal of Computational and Applied Mathematics, Vol. 175, No. 1, pp. 137-147, 2005

[17] G. Psihoyios, T. E. Simos, "A new trigonometrically-fitted sixth algebraic order P-C algorithm for the numerical solution of the radial Schrodinger equation", Mathematical and Computer Modelling, Vol. 42, pp. 887-902, 2005

[18] T. E. Simos, "Dissipative Trigonometrically-fitted methods for linear second-order IVPs with oscillatory solution", Applied Mathematics Letters, Vol. 17, No. 5, pp. 601-607, 2004

[19] G. O. Adejumo, T. O. Abioye, M. Tar, "Adoption of computer assisted language learning software among Nigerian secondary school students", Covenant International Journal of Psychology, Vol. 1, No. 2, pp. 22-30, 2016

[20] A. O. Akinfenwa, S. N. Jator, N. M. Yao, "Continuous block backward differentiation formula for solving stiff ordinary differential equations", Computers and Mathematics with Applications, Vol. 65, No. 7, pp. 9961005, 2013

[21] T. A. Anake, D. O. Awoyemi, A. O. Adesanya, "One-step implicit hybrid block method for the direct solution of general second order ODEs", IAENG International Journal of Applied Mathematics, Vol. 42, No. 4, pp. 224-228, 2012

[22] T. A. Anake, L. O. Adoghe, "A four point integration method for the solutions of IVP in ODE", Australian Journal of Basic and Applied Sciences, Vol. 7, No. 10, pp. 467-473, 2013

[23] J. D. Lambert, Numerical Methods for Ordinary Differential Systems: The Initial Value Problem, John Wiley and Sons, 1991

[24] J. G. Oghonyon, S. A. Okunuga, S. A. Iyase, "Milne's implementation on block predictor-corrector methods", Journal of Applied Sciences, Vol. 16, No. 5, pp. 236-241, 2016

[25] J. G. Oghonyon, S. A. Okunuga, S. A. Bishop, “A Variable-step-size block predictor-corrector method for ODEs”, Asian Journal of Applied Sciences, Vol. 10, pp. 96-101, 2017

[26] Z. B. Ibrahim, K. I. Othman, M. Suleiman, "Variable step block backward differentiation formula for solving first-order stiff ODEs", Proceedings of the World Congress on Engineering, London, U.K, Vol. 2, pp. 2-6, July 2-4, 2007

[27] Z. B. Ibrahim, K. I. Othman, M. Suleiman, "Implicit r-point block backward differentiation formula for solving first-order stiff ODEs", Applied Mathematics and Computation, Vol. 186, No. 1, pp. 558-565, 2007

[28] Z. A. Majid, M. Suleiman, "Parallel direct integration variable step block method for solving large system of higher order ODEs", International Journal of Mathematical and Computational Sciences, Vol. 2, No. 4, pp. 269-273, 2008

[29] S. Mehrkanoon, Z. A. Majid, M. Suleiman, "A variable step implicit block multistep method for solving first-order ODEs", Journal of Computational and Applied Mathematics, Vol. 233, No. 9, pp. 23872394, 2010

[30] Z. A. Majid, M. B. Suleiman, "Implementation of Four-Point Fully Implicit Block Method for Solving Ordinary Differential Equations", Applied Mathematics and Computation, Vol. 184, No. 2, pp. 514-522, 2007 